\title{
Fitting fixed-target and ion collisions in the LHCb Gauss simulation framework
}

\author{
Laure Massacrier ${ }^{1}$, Dmitry Popov ${ }^{2,3}$, Patrick Robbe ${ }^{4, *}$, and Michael Winn ${ }^{5}$ \\ ${ }^{1}$ Institut de Physique Nucléaire d'Orsay(IPNO), Institut National de Physique Nucléaire et de Physique \\ des Particules (IN2P3/CNRS), Université de Paris-Sud, Université Paris-Saclay, Orsay, France \\ ${ }^{2}$ University of Birmingham, Birmingham, United Kingdom \\ ${ }^{3}$ Max-Planck-Institut für Kernphysik (MPIK), Heidelberg, Germany \\ ${ }^{4}$ LAL, Univ. Paris-Sud, CNRS/IN2P3, Université Paris-Saclay, Orsay, France \\ ${ }^{5}$ Université Paris-Saclay Centre d'études de Saclay(CEA), IRFU, Départment de Physique Nucléaire \\ $(\mathrm{DPhN})$, Saclay, France
}

\begin{abstract}
The $\mathrm{LHCb}$ experiment has been operating in various beam configurations in Run 1 and 2 of the LHC, with collisions of lead ion beams or in a fixed-target setup. In order to analyse these data, the Gauss simulation software has been extended to be able to generate events describing these configurations, based mainly on the EPOS event generator. These proceedings give details about the methods employed.
\end{abstract}

\section{Introduction}

The LHCb experiment [1] has recorded since 2013 data in various configurations in addition to the $p p$ collisions on which its main physics program is based. These configurations are collisions of protons with lead ions $(p \mathrm{~Pb})$ at a center-of-mass energy per nucleon pair of $5 \mathrm{TeV}$ in 2013 and 2016, and of 8.16 TeV in 2016, and collisions of lead beams $(\mathrm{PbPb})$ at a center-of-mass energy of $5 \mathrm{TeV}$ in 2015.

Fixed-target collisions are also possible at the $\mathrm{LHCb}$ interaction point, and have been recorded with the detector. They are obtained thanks to the SMOG (System for Measuring Overlap with Gas) device [2, 3], which can inject gas into the LHC vacuum at the $\mathrm{LHCb}$ interaction point. Since 2013, data have been collected with several types of gases: Ne, Ar and $\mathrm{He}$, and both types of LHC beams, $p$ and $\mathrm{Pb}$. In this configuration, the center-of-mass energy of the collision varies between $70 \mathrm{GeV}$ and $110 \mathrm{GeV}$ per nucleon pair.

Several measurements are obtained from the analysis of the recorded data samples, such as a measurement of the $\bar{p}$ production cross-section in $p H e$ collisions at $110 \mathrm{GeV}$ [4], a measurement of the $D^{0}$ and $J / \psi$ production cross-sections in $p H e$ and $p$ Ar collisions at $86.6 \mathrm{GeV}$ and $110.4 \mathrm{GeV}$ [5] or a measurement of the nuclear modification of $J / \psi$ production in $p \mathrm{~Pb}$ collisions at $8.16 \mathrm{TeV}[6]$. These analyses require simulating events in a realistic manner in order to compute efficiencies, with the method described in this document. The framework used for this purpose is the Gauss LHCb simulation framework [7]. The simulation of the detector response is obtained with the Geant 4 toolkit $[8,9]$, as for the simulation of $p p$ collisions. The specificity of the simulation of heavy-ion and fixed-target collisions lies in the

\footnotetext{
*e-mail: robbe@lal.in2p3.fr
} 
generator phase of Gauss, based in this case on the EPOS [10, 11] and Pythia $8[12,13]$ generators and configured specifically to reproduce the features of the experimental conditions.

\section{Simulation of minimum bias heavy-ion or fixed-target collisions in the Gauss framework}

The generation of events uses the same features as for the generation of $p p$ collisions [14]. The sequence is composed of 4 steps described below. Each step corresponds to a generic algorithm which is configured to match the heavy-ion or fixed-target behaviour via Python steering files. These generic algorithms are originally developed for the generation of $p p$ collisions in Gauss and can be re-used without modification.

\subsection{Generation of the beam properties}

The beam properties are generated according to the ones of the LHC at the time of the collisions that are simulated. The parameters comprise the beam energy, the type of particles in the beam or in the target ( $p, \mathrm{~Pb}$ or the type of gas), and the direction of the LHC beam. This way, the non-zero angle of the beam with respect to the LHCb detector axis is correctly taken into account. The directions of the beams are smeared according to the values of the emittance and of the $\beta^{*}$ function.

\subsection{Generation of the interaction region}

For colliding beams, the interaction region is generated according to a Gaussian distribution along the three axes, $x, y$ and $z$ with mean values and sizes equal to the values measured during the data taking periods. In the case of fixed target collisions, the gas pressure is assumed uniform in a region of $50 \mathrm{~cm}$ length along the $z$ axis and centered on the interaction point. This assumption neglects a variation of the pressure of $3 \%$ observed in data. This region corresponds typically to the events selected for the analyses. The collisions are generated uniformly along $z$ and with Gaussian shapes in the transverse directions, as for colliding beams.

\subsection{Generation of one minimum-bias interaction}

A minimum bias interaction is generated using the EPOS generator, configured as described in Section 4. The EPOS generator, as interfaced to the Gauss software, generates events in the center-of-mass frame of the collisions. The event obtained is then boosted to the laboratory frame. This ensures also that the angle between the beams is correctly simulated.

\subsection{Decay of the unstable particles}

The EPOS generator is configured in such a way that the particles produced are all considered stable, even the ones which are in reality not stable (for example, $\pi^{0}$ mesons or $\Delta$ baryons). The generation of the decay of these particles is done separately by the EvtGen generator [15]. EvtGen is configured with a detailed decay table and can thus precisely describe the final states of the decays and their kinematics. The events obtained at the end of this sequence are processed by Geant4 in order to simulate complete events. 


\section{Simulation of signal events}

The LHCb simulation framework offers the possibility to generate "signal" event samples. The samples contain in each event a signal decay of interest for a given analysis, for example the decay $J / \psi \rightarrow \mu^{+} \mu^{-}$where the decay is forced to a pre-defined final state. The signal samples contain also the other particles produced in the collision, in addition to the final state particles coming from the signal decay. They are obtained embedding a signal decay generated by Pythia 8 in a minimum bias interaction generated by EPOS, as explained below. This allows an efficient analysis of events with small probabilities to occur in minimum bias samples.

Signal particles are produced by the Pythia 8 generator. This generator is configured to generate $p p$ minimum bias events at a center-of-mass energy equal to the center-of-mass energy per nucleon pair of the studied heavy-ion or fixed-target collision. Using a minimum bias configuration allows one to simulate the production of the signal particles from all possible processes. For example, in the case of signal $J / \psi$ generation, it includes production of $J / \psi$ coming from the decays of $b$ hadrons in addition to the direct production of $J / \psi$ mesons from the parton-parton interaction. The configuration of the Pythia generator also takes into account the angles between the beams and the position of the interaction as described in the previous section.

Once an event with the particle of interest $(J / \psi$ for the example given above) is obtained amongst the minimum bias interactions, the EvtGen generator is called to decay this particle into the signal final state $\left(J / \psi \rightarrow \mu^{+} \mu^{-}\right.$in the example). Then all other particles that were generated by Pythia together with the signal particle are removed, and only the signal decay chain is kept. This decay chain is merged with a minimum bias interaction generated by the EPOS generator following the sequence detailed in the previous section.

One should note that the correlation between the signal production cross-section and the number of participants in the interaction is ignored with this method. In reality the probability to find the signal particle is proportional to the number of participants in the collision (assuming no nuclear matter effect) because the production cross-section is proportional to the number of participants. Signal events should then have a particle multiplicity, on average, larger than minimum bias events because the particle multiplicity is also proportional to the number of participants in the collision. With the method described here, this is not the case because the signal samples and the minimum bias samples are generated with the same distribution of the number of participants, hence with the same average particle multiplicity. The bias introduced is corrected at the analysis level, weighting the simulated signal events according to their particle multiplicity in order to reproduce the multiplicity observed in data.

The generation of signal events is configured using a Python option file and an EvtGen decay file. They specify the type of particle and the final state. These files are generic and have a syntax which is independent of the collision system. They can thus be used indifferently for the simulation of $p p$, heavy-ion or fixed-target collisions.

\section{Interface to the EPOS generator}

The EPOS generator is interfaced to the Gauss software so that it can be used easily in the sequence described in Section 2. The version used in the LHCb software is the version available from the CRMC distribution [11]. The EPOS implementation is configured to use the "EPOS LHC" model, a minimum impact parameter of 0 and a maximum impact parameter of $20 \mathrm{fm}$, except for the simulation of $\mathrm{PbPb}$ collisions where the range is restricted between $8 \mathrm{fm}$ and $22 \mathrm{fm}$. The decays of hadrons inside EPOS are switched off, and all particle masses 
and widths are updated with the values used in the LHCb software. All other parameters are taken from the default configuration of EPOS.

\section{Conclusions}

Thanks to the flexibility of its design, the Gauss simulation framework is extended to include the possibility to simulate with great details, collisions of heavy-ion or fixed-target events as recorded by the LHCb experiment. This setup has been used for several published measurements. Other dedicated heavy-ion generators will be integrated following the same logic in the future to improve the capability of the simulation software to describe the variety of physics processes studied by the LHCb collaboration. In particular, the usage of Pythia 8 to generate heavy-ion interactions according to the Angantyr model [16] is currently under investigation.

\section{References}

[1] LHCb collaboration, A. A. Alves Jr. et al., JINST 3 (2008) S08005.

[2] C. Barschel, Precision luminosity measurement at LHCb with beam-gas imaging, $\mathrm{PhD}$ thesis, RWTH Aachen U., 2014, CERN-THESIS-2013-301.

[3] LHCb collaboration, R. Aaij et al., JINST 9 (2014) P12005, arXiv:1410.0149.

[4] LHCb collaboration, R. Aaij et al., arXiv:1808.06127

[5] LHCb collaboration, R. Aaij et al., arXiv:1810.07907.

[6] LHCb collaboration, R. Aaij et al., Phys. Lett. B774 (2017) 159, arXiv:1706.07122.

[7] M. Clemencic et al., J. Phys. Conf. Ser. 331 (2011) 032023.

[8] Geant4 collaboration, J. Allison et al., IEEE Trans. Nucl. Sci. 53 (2006) 270.

[9] Geant4 collaboration, S. Agostinelli et al., Nucl. Instrum. Meth. A506 (2003) 250.

[10] T. Pierog et al., Phys. Rev. C92 (2015) 034906, arXiv:1306.0121.

[11] T. Pierog, C. Baus, and R. Ulrich, https://web.ikp.kit.edu/rulrich/crmc.html.

[12] T. Sjöstrand, S. Mrenna, and P. Skands, Comput. Phys. Commun. 178 (2008) 852, arXiv:0710.3820.

[13] T. Sjöstrand, S. Mrenna, and P. Skands, JHEP 05 (2006) 026, arXiv:hep-ph/0603175.

[14] I. Belyaev et al., J. Phys. Conf. Ser. 331 (2011) 032047.

[15] D. J. Lange, Nucl. Instrum. Meth. A462 (2001) 152.

[16] C. Bierlich et al., JHEP 10 (2018) 134, arXiv:1806.10820. 